\title{
Peripapillary retinal nerve fiber layer and ganglion cell complex degeneration in Egyptian patients with bipolar disorder
}

\author{
Dalia H. Khalil ${ }^{1}$ Mohamed M. Said ${ }^{2}$
}

Received: 21 May 2017 / Revised: 20 February 2019 / Accepted: 5 March 2019 / Published online: 29 March 2019

(c) The Royal College of Ophthalmologists 2019

\begin{abstract}
Purpose To determine if changes in OCT parameters including peripapillary RNFL thickness and GCC thickness in Egyptian patients with bipolar disorder exist, and to correlate them with disease severity and clinical characteristics.

Methods A case-control study conducted on 40 patients with bipolar disorder were compared to 40 matched healthy controls. Both patients and controls were subjected to ophthalmic examination including: BCVA, slit-lamp examination, fundus examination, and OCT imaging. Patients were also subjected to Young Mania Rating Scale (YMRS).

Results Thinning of average (RNFL) and average (GCC) thickness of right and left eye in patients with BPD (whether received electroconvulsive therapy or not) when compared with control subjects. Number of episodes, age at onset and severity of disease showed insignificant correlation with OCT parameters.

Conclusions Significant degenerative changes were detected by OCT in patients with bipolar disorder that were not affected by receiving ECT and not related to disease severity or duration.
\end{abstract}

\section{Introduction}

Bipolar disorder (BPD) is a chronic episodic psychiatric disorder that has a prevalence of $1 \%$ worldwide of general population with equal male to female distribution [1]. Magnetic resonance imaging (MRI) in bipolar disorder suggests the presence of structural brain abnormalities, which are detected in patients starting from their first episode. Some progressive changes are found, such as reduction of brain gray matter in fusiform gyrus, hippocampus and also in the temporal lobe. Severity of changes can be related to symptoms severity and episodes number [2, 3]. These structural changes may support the neurodegenerative nature of bipolar disorder [4].

These authors contributed equally: Dalia H. Khalil, Mohamed M. Said

Dalia H. Khalil

Daliahamed_76@yahoo.com

1 Ophthalmology Department, Faculty of Medicine, Cairo University, Giza, Egypt

2 Ophthalmology Department, Faculty of Medicine, Fayoum University, Faiyum, Egypt
Optical coherence tomography (OCT) is a non-invasive imaging technique that allows in vivo visualization of different retinal layers. Retinal nerve fiber layer (RNFL) is made up of non myelinated axons of the ganglion cells so it represents an objective and sensitive parameter for evaluating degeneration in neurological and psychiatric disorders like multiple sclerosis, schizophrenia, and bipolar disorder [5]. Whereas, the ganglion cell complex (GCC) represents the three innermost retinal layers: the nerve fiber layer, the ganglion cell layer, and the inner plexiform layer. Therefore, it is considered a part of the central nervous system and can also be used in quantification of neurodegenerative process in the retina. OCT is easy to perform, inexpensive, and highly reproducible [6].

The aim of this study was to determine if there are differences in OCT parameters in Egyptian patients with BPD compared to matched controls, and to see if they correlate with disease severity and clinical characteristics.

\section{Patients and methods}

This is a case-control observational cross-sectional study comparing two groups. The first group enrolled patients who have bipolar disorder, while the second group included psychiatric disease free subjects. Psychiatric diagnoses were 
excluded in control subjects-who were selected from paramedical personnel working in Kasr Al-Ainy Hospitals -by using Structured Clinical Interview for DSM-IV (SCID IV) [7].

\section{Inclusion criteria}

Diagnosed patients with bipolar (I) disorder confirmed with the SCID IV without psychotic features. Patients were selected consecutively-convenient sample-from those who were admitted in the inpatient unit of Kasr Al-Ainy Psychiatry and Addiction Hospital starting from April 2016 to June 2016. Patients with any type of episode-manic, mixed, or depressive-were included regardless whether they were in episode or in partial remission.

Using medications or electroconvulsive therapy did not exclude patients from the study.

\section{Exclusion criteria}

Medical disorders that may affect the OCT findings (including hypertension, diabetes mellitus, glaucoma, increased intracranial tension, and multiple sclerosis) were excluded in both groups by history taking.

The study was approved by the Research Ethics Committee of Faculty of Medicine, Cairo University (N-4-2016) and was conducted in accordance to the ethical principles of the Declaration of Helsinki. Patients who agreed to participate in the study were asked to sign an informed written consent that explained all required steps of the study.

The patients were subjected to:

\section{(1) Optical coherence tomography (OCT)}

Performed in the Kasr Al-Ainy Laser Unit of the Ophthalmology Department in the Kasr Al-Ainy hospitals using the RTVue spectral-domain OCT (Optovue Inc, Fremont, CA) based on Fourier-domain optical coherence technology.

Both eyes in each patient were examined and the procedure was completed in about 5-10 min.

Clinical assessment of patients including: best corrected visual acuity (BCVA), slit-lamp examination, and fundus examination were done in all cases prior to OCT and the research personnel who conducted it were blind to the diagnosis of the patients.

\section{Protocols}

(1) Macular map protocol as a fast scan for GCC measurement, including 12 radial line scans (1024 A-scans per line) in a 3-dimensional $6 \times 6 \mathrm{~mm}$ area (2.0 s). (2) The peripapillary RNFL NHM4 protocol that included 12 radial scans (452 Ascans per line) over $3.45 \mathrm{~mm}$ diameter, centered on the optic disc. It allowed eye tracking, signal noise reduction, and inner nuclear layer (INL) observation for the presence of macular microcysts. Scans were performed with ambient lighting and without pupil dilation decreasing subjective distress. OSCARIB protocol [8] was used to assure quality of OCT.

Subjects were asked to position their heads in the head mount and to fix on a target light either inside the ocular lens (internal fixation) or outside to the ocular lens (external fixation).

If the internal fixation method is selected, the patient's fellow eye is covered while scanning. This enabled the patient to fixate more steadily.

\section{(2) Psychiatric assessment}

Both groups were assessed by the Structured Clinical Interview for DSM-IV (SCID IV) as mentioned above. The patient group had gone further assessment using Young Mania Rating Scale (YMRS) and 17-Item Hamilton Depression Rating Scale (HAM-D).

Demographic data, clinical details, and patients' psychiatry, and medical histories were all assessed using the semi structured clinical interview of Kasr Al-Ainy Psychiatry Hospital.

YMRS is a rating scale used to evaluate manic symptoms at baseline and over time in individuals with mania [9]. The HAM-D was used to rate the severity of depression in patients [10]. Both tests were performed for all patients regardless of the type of episode within $24 \mathrm{~h}$ from performing the OCT measurement.

\section{Statistical analysis}

Sample size calculation was done using G*Power software version 3.1.9.2. The effect size estimated from a previous study was $0.8, \alpha=0.05$ and power $=0.9$. The required sample size was 35 for each group [11].

Results were evaluated statistically by the Statistical Package for the Social Sciences (SPSS) version 20 (IBM, 2011). Normality of data was tested by KolmogorovSmirnov test. To describe the data, frequency (percent), and mean $\pm \mathrm{SD}$ were used. $T$-test and Pearson correlation test were used for comparisons and correlations respectively. $P$-values $<0.05$ were considered statistically significant and $95 \%$ confidence interval $(\mathrm{CI})$ was calculated.

\section{Results}

Forty patients were included in the study - their ages ranged from 18- to 55-years-old with mean age of $30.90 \pm 9.31$ of both sexes. In all, $52.5 \%$ of patients were males and $47.5 \%$ were females Majority of patients were single $(52.5 \%)$ and 
Table 1 Comparison between patients and controls with regard to age, sex, and RNFL thickness

Table 2 Comparison between patients and controls with regard to GCC thickness

Fig. 1 Average RNFL \& GCC thickness in bipolar cases in relation to normal subjects

\begin{tabular}{|c|c|c|c|c|c|}
\hline & \multirow{2}{*}{$\begin{array}{l}\text { Patients } \\
N: 40 \\
\text { Mean } \pm \text { SD }\end{array}$} & $\begin{array}{l}\text { Controls } \\
N: 40\end{array}$ & \multicolumn{2}{|c|}{$\begin{array}{l}95 \% \text { Confidence } \\
\text { interval }\end{array}$} & \multirow[t]{2}{*}{$P$-value } \\
\hline & & Mean & Lower & Upper & \\
\hline Age (years) & $30.90 \pm 9.31$ & $32.85 \pm 8.77$ & -2.08 & 5.98 & 0.338 \\
\hline Sex (male:female) & $21: 19$ & $23: 17$ & - & - & 0.653 \\
\hline Right average RNFL & $104.24 \pm 7.63$ & $121.35 \pm 3.98$ & 14.39 & 19.84 & $<0.001^{\mathrm{a}}$ \\
\hline Left average RNFL & $104.42 \pm 9.35$ & $120.65 \pm 3.70$ & 13.03 & 19.42 & $<0.001^{\mathrm{a}}$ \\
\hline Right superior RNFL & $104.07 \pm 9.06$ & $116.82 \pm 21.80$ & 5.25 & 20.23 & $0.001^{\mathrm{a}}$ \\
\hline Left superior RNFL & $106.00 \pm 11.69$ & $118.42 \pm 21.62$ & 4.64 & 20.19 & $0.002^{\mathrm{a}}$ \\
\hline Right inferior RNFL & $106.49 \pm 10.94$ & $115.77 \pm 20.02$ & 2.06 & 16.49 & $0.013^{\mathrm{a}}$ \\
\hline Left inferior RNFL & $105.35 \pm 10.07$ & $116.64 \pm 21.69$ & 3.72 & 18.87 & $0.004^{\mathrm{a}}$ \\
\hline Right nasal RNFL & $80.32 \pm 9.15$ & $84.20 \pm 10.49$ & -0.52 & 8.26 & 0.082 \\
\hline Left nasal RNFL & $82.25 \pm 9.49$ & $84.37 \pm 7.12$ & -1.61 & 5.86 & 0.262 \\
\hline Right temporal RNFL & $83.14 \pm 9.78$ & $89.27 \pm 9.23$ & 1.90 & 10.37 & $0.005^{\mathrm{a}}$ \\
\hline Left temporal RNFL & $79.76 \pm 10.48$ & $86.10 \pm 9.93$ & 1.79 & 10.89 & $0.007^{\mathrm{a}}$ \\
\hline
\end{tabular}

$R N F L$ retinal nerve fiber layer

${ }^{a} P$-value $<0.05$ is considered statistically significant

\begin{tabular}{llllll}
\hline & Patients & Controls & \multicolumn{2}{l}{ Confidence interval } & \multirow{2}{*}{$P$-value } \\
\cline { 5 - 6 } & $N: 40$ & $N: 40$ & Upper & Lower & \\
\hline Right average GCC & $94.69 \pm 7.00$ & $100.75 \pm 9.53$ & 2.33 & 9.78 & $0.002^{\mathrm{a}}$ \\
Left average GCC & $93.71 \pm 6.68$ & $101.76 \pm 9.17$ & 4.47 & 11.63 & $<0.001^{\mathrm{a}}$ \\
Right superior GCC & $94.71 \pm 6.79$ & $97.96 \pm 8.60$ & -0.20 & 6.70 & 0.064 \\
Left superior GCC & $93.52 \pm 6.25$ & $99.38 \pm 7.56$ & 2.77 & 8.95 & $<0.001^{\mathrm{a}}$ \\
Right inferior GCC & $94.66 \pm 7.65$ & $98.00 \pm 7.69$ & -0.08 & 6.75 & 0.055 \\
Left inferior GCC & $94.32 \pm 7.59$ & $98.42 \pm 6.79$ & 0.89 & 7.30 & $0.013^{\mathrm{a}}$ \\
\hline
\end{tabular}

GCC ganglion cell complex

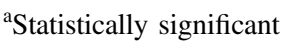

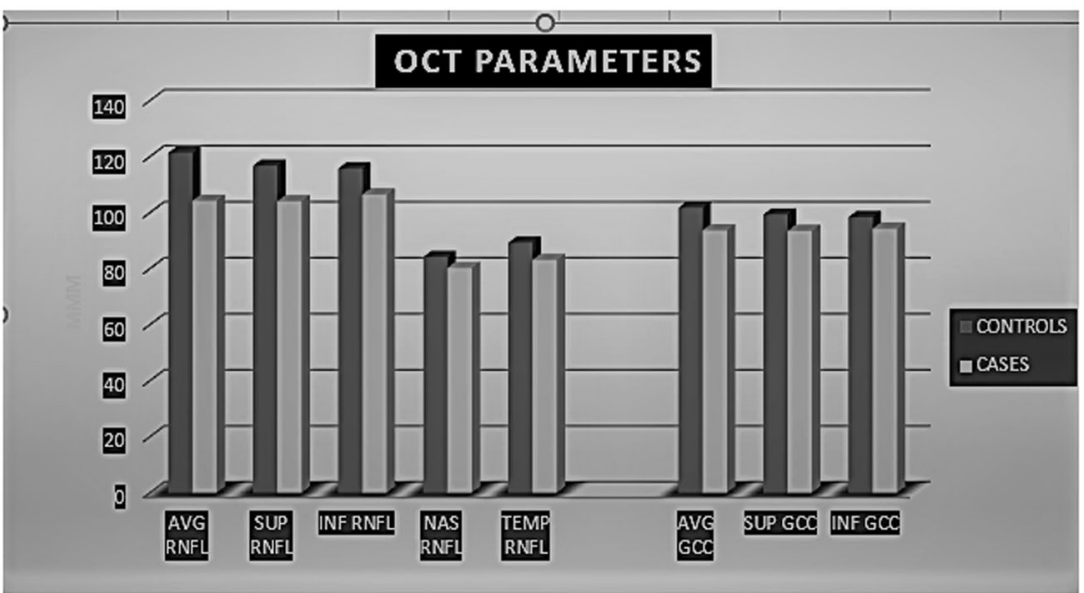

$(40 \%)$ were not working, $15 \%$ of patients had received electroconvulsive therapy (ECT) within the last year. The control group included 40 age- and sex-matched, psychiatric disease free subjects. There was no difference between patients and control subjects in age or gender.
Patients showed thinning of peripapillary RNFL thickness in relation to control subjects in most of the OCT parameters except for right and left nasal; they did not show significant difference (Table 1). They also showed significant decrease in GCC thickness with regard to right and 
Table 3 Patients parameters with regard to gender difference
Male patients $(n=21)$ Mean \pm SD $\quad$ Female patients $(n=19) \quad P$-value Mean \pm SD

\begin{tabular}{lccc}
\hline Age (years) & $28.57 \pm 7.10$ & $33.47 \pm 10.88$ & 0.106 \\
Age of onset & $21.56 \pm 5.45$ & $26.21 \pm 8.90$ & 0.058 \\
HAM-D $^{\mathrm{a}}$ & $6.00 \pm 2.47$ & $10.56 \pm 8.25$ & $\mathbf{0 . 0 3 1 ^ { * }}$ \\
YMRS $^{\mathrm{b}}$ & $18.86 \pm 9.65$ & $14.77 \pm 10.48$ & 0.207 \\
Right superior $_{\text {RNFL }}^{\mathrm{c}}$ & $100.85 \pm 8.15$ & $107.63 \pm 8.86$ & $\mathbf{0 . 0 1 6}^{*}$ \\
Left superior RNFL & $105.69 \pm 12.25$ & $106.35 \pm 11.35$ & 0.862 \\
Right average GCC & $96.81 \pm 7.31$ & $92.34 \pm 5.97$ & $\mathbf{0 . 0 4 2}$ \\
Left average GCC & $94.39 \pm 5.72$ & $92.96 \pm 7.70$ & 0.507 \\
Right superior GCC & $96.99 \pm 6.75$ & $92.20 \pm 6.04$ & $\mathbf{0 . 0 2 4}$ \\
\hline
\end{tabular}

$P$-value $<0.05$ is considered significant

${ }^{\mathrm{a}} H A M-D$ Hamilton depression

${ }^{\mathrm{b}} Y M R S$ Young Mania Rating Scale

${ }^{\mathrm{c}} R N F L$ retinal nerve fiber layer

${ }^{\mathrm{d}} G C C$ ganglion cell complex

Bold values with $(*)$ indicate significance

\begin{tabular}{|c|c|c|c|c|c|}
\hline & \multirow[t]{2}{*}{$\begin{array}{l}\text { Right eyes in patients } N: 40 \\
\text { Mean } \pm \text { SD }\end{array}$} & \multirow[t]{2}{*}{$\begin{array}{l}\text { Left eyes in patients } N: 40 \\
\text { Mean } \pm \mathrm{SD}\end{array}$} & \multicolumn{2}{|c|}{$\begin{array}{l}95 \% \text { confidence } \\
\text { interval }\end{array}$} & \multirow[t]{2}{*}{$P$-value } \\
\hline & & & Lower & Upper & \\
\hline Average $\mathrm{RNFL}^{\mathrm{a}}$ & $104.24 \pm 7.63$ & $104.42 \pm 9.35$ & -3.98 & 3.61 & 0.924 \\
\hline Superior RNFL & $104.07 \pm 9.06$ & $106.00 \pm 11.69$ & -6.58 & 2.72 & 0.412 \\
\hline Inferior RNFL & $106.49 \pm 10.94$ & $105.35 \pm 10.07$ & -3.53 & 5.82 & 0.628 \\
\hline Nasal RNFL & $80.32 \pm 9.15$ & $82.25 \pm 9.49$ & -6.07 & 2.22 & 0.358 \\
\hline Temporal RNFL & $83.13 \pm 9.77$ & $79.76 \pm 10.48$ & -1.13 & 7.89 & 0.140 \\
\hline Average $\mathrm{GCC}^{\mathrm{b}}$ & $94.68 \pm 6.99$ & $93.71 \pm 6.68$ & -2.06 & 4.02 & 0.525 \\
\hline Superior GCC & $94.71 \pm 6.78$ & $93.52 \pm 6.24$ & -1.71 & 4.09 & 0.417 \\
\hline Inferior GCC & $94.66 \pm 7.65$ & $94.32 \pm 7.59$ & -3.05 & 3.73 & 0.843 \\
\hline
\end{tabular}

$P<0.05$ is considered significant

${ }^{\mathrm{a}} R N F L$ retinal nerve fiber layer

${ }^{\mathrm{b}} G C C$ ganglion cell complex
Table 4 Comparison between right and left eyes of the patients with regard to optical coherence tomography (OCT) measures left average, left superior and inferior; While the right superior and inferior were not significant (Table 2).

Figure 1 shows the average values of RNFL and GCC of bipolar cases in relation to normal subjects.

Male and female patients were not different in age, age of onset, or YMRS scores while females had higher scores on HAM-D. OCT parameters did not differ significantly between the two genders except in right superior RNFL thickness, right average, and superior GCC thickness (Table 3). Comparison between the two eyes in all OCT parameters revealed no significant differences (Table 4).

Only the right inferior peripapillary RNFL thickness showed positive correlation with the number of manic episodes $(P=0.034)$.

Comparison between patients who received previous (ECT) and those who didnot showed statistically insignificant differences with regard to peripapillary RNFL thickness and GCC thickness.

With regard to age of onset, number of depressive episodes, number of mixed episodes, total number of episodes, or number of episodes per year no significant correlation was detected with peripapillary RNFL thickness or GCC thickness. Similarly, age of patients, scores of YMRS, and HAM-D had no significant correlation with OCT parameters.

\section{Discussion}

Bipolar disorder is a multifactorial chronic mental disorder. Different neuroimaging methods have been frequently used by researchers to identify specific abnormalities associated 
with this disorder. However, there are no clearly defined pathognomonic findings to this disorder [12].

While, some studies have demonstrated signs of nervous system degeneration in patients with first episode BPD [3], other studies have linked these findings to the longitudinal course of illness and severity of mood episodes [13]. Whether these signs of degeneration are state or trait markers of illness continues to be a debatable issue.

This study aimed at proving optical nerve degenerative changes in patients with BPD. To test this hypothesis, we compared bipolar patients to age- and sex-matched group of control subjects; we correlated OCT parameters with clinical variables in the patient group. The main significant finding was the deceased, most of the OCT parameters in patients compared to control subjects.

There was a difference between patients and controls, but we did not find a correlation between OCT and clinical parameters.

Patients had decreased thickness of all RNFL parameters with significant differences between patients and controls in average, superior, inferior, and temporal RNFL. Mehraban et al. [11] found similar results between bipolar patients and controls. Other illness parameters like age at onset of BPD, number of manic and/or depressive episodes were not correlated, which was explained by the fact that $86.7 \%$ of his patients suffered only a single manic episode.

On the other hand, Kalenderoglu et al. [14] reported decrease in RNFL thickness but not significant from controls. The GCC volume was also significantly lower in the patient group. There was a significant negative correlation between the disease parameters such as the disease duration, YMRS score, clinical global impression scale, number of hospitalizations, and GCC volume although the patients were euthymic at the time of the study. The study found that the GCC volume loss was much more prominent than the decrease in the RNFL thickness and suggested that neurodegeneration in bipolar disorder starts in the GCC, which forms neuronal cell bodies, and then gradually progresses to axonal degeneration. A significant correlation between GCC loss and disease duration also suggested progressive degeneration or inflammation. The contradiction between these results would raise a debate about whether the RNFL thinning is related to the severity of mood symptoms or not. To test this idea, we correlated the RNFL scores and both YMRS and HDRS in our study, however, we failed to find such association.

Another study investigating OCT parameters in patients with Major Depression found that depressed patients were not different from the healthy controls with regard to OCT parameters. However, IPL (inner plexiform layer) and nasal RNFL were negatively correlated with the duration of the latest depressive episode [15].
A recent study designed to compare retinal OCT measures of patients with schizophrenia and controls and evaluate possible differences between recent illness episode (RIE) and non-recent illness episode (NRIE) patients. The study found significantly reduced RNFL overall measures, superior RNFL, nasal RNFL, macular volume, and macular inner ring thickness in schizophrenic patients when compared to healthy controls. However, no significant correlation was found between illness duration and retinal measures. The study concluded that retinal parameters observed using OCT in schizophrenia were not related to clinical status and this limits its potential role as a state biomarker of the disorder [16].

In 2016, Cunha et al. [17] demonstrated significant reduction in the peripapillary RNFL thickness in $\mathrm{AD}$ patients, suggesting diffuse axonal degeneration. Their finding was confirmed by macular thickness reduction and ganglion cell layer impairment in AD patients.

In the same year, Pillai et al. [18] found insignificant difference between patients with alzheimer's disease (AD) dementia, and age- and sex-matched controls with regard to RNFL thickness, GCC thickness, and macular volume (MV).

In 2017, Ferrari et al. [19] confirmed the presence of retinal neuroaxonal thinning in $\mathrm{AD}$ cases that correlated with disease severity and they suggested that retinal and brain neurodegeneration may occur in parallel to some extent.

In 2018, Poroy and Yücel [20] demonstrated significantly higher foveal thickness and volume in $\mathrm{AD}$ patients than controls, but they didnot find significant difference with regard to peripapillary RNFL.

According to our results, there were no gender or agerelated differences detected in OCT measurements. To our knowledge, there are no published studies to date comparing OCT measurements among male and female or across different age groups in patients with bipolar disorder. In normal population, contradictory results were found. Alasil et al. [21] reported significant decrease in thickness of RNFL with age, but no differences were reported between males and females. Male RNFL was significantly thicker than female RNFL in another study [22]. A third study showed different age and gender effects in different retinal areas [23]. The contradiction between these studies may be related to the ethnic difference, low number of included subjects, and the sensitivity of the used devices. The effect of bipolar disorder on these differences could not be confirmed.

Most of the published studies didnot show differences in most of the OCT parameters between the right and the left eye except for a single study that found a statistically significant difference between right and left eyes for the mean RNFL thickness in normal population [22]. 
In conclusion, patients with bipolar disorder show thinning of RNFL and GCC detected by OCT and these changes were not affected by receiving ECT and not related to severity or duration of illness. Our results also confirm the role of nervous system degeneration in terms of gray matter loss in patients with bipolar disorder. The failure to find a relation between the OCT changes and both severity and duration of illness may increase indecision about the etiology of the illness, whether developmental or degenerative?

Further studies including larger sample size, first degree relatives of bipolar patients and individuals at risk of developing bipolar disorder may demonstrate the role of OCT as a biomarker in bipolar disorder and confirm the neurodevelopmental or neurodegenerative nature of the illness.

\section{Limitations}

Limitations of the study include: the small sample size, the cross-sectional design that precluded the examination of causality, the effect of atypical anti-psychotic, mood stabilizing medications, and smoking that was not ruled out. Valproate was found to cause gray matter loss in patients suffering from epilepsy while lithium was found to have a neuroprotective role in BP patients.

Finally, we confirm that this study was self-funded by the researchers and did not receive any fund from pharmaceutical companies or any other funding agent.

\section{Summary}

\section{What was known before}

- Bipolar disorder may affect ocular structures

\section{What this study adds}

- Determine if there are changes in OCT parameters, including peripapillary RNFL thickness and GCC thickness in Egyptian patients with bipolar disorder.

\section{Compliance with ethical standards}

Conflict of interest The authors declare that they have no conflict of interest.

Publisher's note: Springer Nature remains neutral with regard to jurisdictional claims in published maps and institutional affiliations.

\section{References}

1. Merikangas KR, Akiskal HS, Angst J, Greenberg PE, Hirschfeld RM, Petukhova M, et al. Lifetime and 12-month prevalence of bipolar spectrum disorder in the National Comorbidity Survey replication. Arch Gen Psychiatry. 2007;64:543-52.

2. Moorhead TW, Mckirdy J, Sussmann JE, Hall J, Lawrie SM, Johnstone EC, et al. Progressive gray matter loss in patients with bipolar disorder. Biol Psychiatry. 2007;62:894-900.

3. Vita A, De Peri L, Sacchetti E. Gray matter, white matter, brain, and intracranial volumes in first-episode bipolar disorder: a metaanalysis of magnetic resonance imaging studies. Bipolar Disord. 2009;11:807-14.

4. Kempton MJ, Geddes JR, Ettinger U, Williams SC, Grasby PM. Meta-analysis, database, and meta-regression of 98 structural imaging studies in bipolar disorder. Arch Gen Psychiatry. 2008;65:1017-32.

5. Bock M, Paul F, Dorr J. [Diagnosis and monitoring of multiple sclerosis: the value of optical coherence tomography]. Nervenarzt. 2013;84:483-92.

6. Khalil DH, Said MM, Abdelhakim MA, Labeeb DM. OCT and visual field changes as useful markers for follow-up of axonal loss in multiple sclerosis in Egyptian patients. Ocul Immunol Inflamm. 2016;25:1-8.

7. First MB, Williams Janet BW, Spitzer RL, Gibbon M. Structured clinical interview for DSM-IV-TR axis I disorders, clinical trials version (SCID-CT). New York: Biometrics Research, New York State Psychiatric Institute; 2017.

8. Tewarie P, Balk L, Costello F, Green A, Martin R, Schippling S, et al. The OSCAR-IB consensus criteria for retinal OCT quality assessment. PLoS ONE. 2012;7:e34823. https://doi.org/10.1371/ journal.pone.0034823.

9. Young RC, Biggs JT, Ziegler VE, Meyer DA. A rating scale for mania: reliability, validity and sensitivity. $\mathrm{Br} \mathrm{J}$ Psychiatry. 1978;133:429-35.

10. Hamilton M. A rating scale for depression. J Neurol Neurosurg Psychiatry. 1960;23:56-62.

11. Mehraban A, Samimi SM, Entezari M, Seifi MH, Nazari M, Yaseri M. Peripapillary retinal nerve fiber layer thickness in bipolar disorder. Graefes Arch Clin Exp Ophthalmol. 2016;254:365-71.

12. Ayhan Y, Sen Z. Neurobiology of bipolar affective disorder: Recent findings on cell and animal studies. Turk Klin J Psychiatry-Spec Top. 2013;6:14-24.

13. Frey BN, Zunta-Soares GB, Caetano SC, Nicoletti MA, Hatch JP, Brambilla $\mathrm{P}$, et al. Illness duration and total brain gray matter in bipolar disorder: evidence for neurodegeneration? EurNeuropsychopharmacol. 2008;18:717-22.

14. Kalenderoglu A, Sevgi-Karadag A, Celik M, Egilmez OB, HanAlmis B, Ozen ME. Can the retinal ganglion cell layer (GCL) volume be a new marker to detect neurodegeneration in bipolar disorder? Compr Psychiatry. 2016;67:66-72.

15. Yildız M, Alim S, Batmaz S, Demir S, Songur E, Ortak H, et al. Duration of the depressive episode is correlated with ganglion cell inner plexifrom layer and nasal retinal fiber layer thicknesses: Optical coherence tomography findings in major depression. Psychiatry Res: Neuroimaging. 2016;251:60-6.

16. Celik M, Kalenderoglu A, Sevgi Karadag A, Bekir Egilmez O, Han-Almis B, Simsek A. Decreases in ganglion cell layer and inner plexiform layer volumes correlate better with disease severity in schizophrenia patients than retinal nerve fiber layer thickness: Findings from spectral optic coherence tomography. Eur Psychiatry. 2016;32:9-15.

17. Cunha LP, Almeida ALM, Costa-Conha LVF, Costa CF, Monteiro MLR. The role of optical coherence tomography in Alzheimer's disease. Int J Retin Vitr. 2016;2-24. https://doi.org/10.1186/ s40942-016-0049-4. 
18. Pillai JA, Bermel R, Bonner-Jackson A, Rae-Grant A, Fernandez $\mathrm{H}$, Bena J, et al. Retinal nerve fiber layer thinning in Alzheimer's disease: A case-control study in comparison to normal aging, parkinson's disease, and non-alzheimer's dementia. Am J Alzheimers Dis Other Demen. 2016;31:430-6.

19. Ferrari L, Huang SC, Magnani G, Ambrosi A, Comi G, Leocani L. Optical coherence tomography reveals retinal neuroaxonal thinning in frontotemporal dementia as in Alzheimer's disease. J Alzheimers Dis. 2017;56:1101-7.

20. Poroy C, Yücel AÂ. Optical coherence tomography: Is really a new biomarker for Alzheimer's disease? Ann Indian Acad Neurol Apr-Jun. 2018;21:119-25.
21. Alasil $\mathrm{T}$, Wang $\mathrm{K}$, Keane $\mathrm{PA}$, Lee $\mathrm{H}$, Baniasadi $\mathrm{N}$, De Boer JF, et al. Analysis of normal retinal nerve fiber layer thickness by age, sex, and race using spectral domain optical coherence tomography. J Glaucoma. 2013;22: 532-41.

22. Sani RY, Abdu L, Pam V. Retinal nerve fiber layer thickness measurements of normal Northern Nigerian adults using optical coherence tomography. Ann Afr Med. 2016;15:52-7.

23. Appukuttan B, Giridhar A, Gopalakrishnan M, Sivaprasad S. Normative spectral domain optical coherence tomography data on macular and retinal nerve fiber layer thickness in Indians. Indian J Ophthalmol. 2014;62:316-21. 